\title{
Extracción de Mo(VI) de disoluciones en medio nítrico mediante Alamine 336 o Aliquat $336^{(*)}$
}

\author{
P. Navarro ${ }^{(*)}$ y F.J. Alguacil ${ }^{(* *)}$ \\ Resumen El tratamiento de concentrados de molibdenita mediante ácido nítrico da lugar a disoluciones en las \\ que, junto al molibdeno aparecen el cobre y el hierro como impurezas. El presente trabajo estudia la \\ purificación de dichas disoluciones empleando la amina terciaria Alamine 336, la sal de amonio cua- \\ ternario Aliquat 336 o mezclas de ambos compuestos como agentes de extracción selectivos del \\ molibdeno. La etapa de reextracción se lleva a cabo con disoluciones de hidróxido amónico, obte- \\ niéndose disoluciones de molibdato amónico que se pueden recircular al circuito principal de obten- \\ ción del mismo. \\ Palabras clave: Extracción con disolventes. Purificación. Molibdeno. Aminas. Sales de amonio \\ cuaternario.

\section{Extraction of Mo(VI) solutions in nitric media by Alamine 336 or Aliquat 336}

\begin{abstract}
The treatment of molybdenite concentrates with nitric acid yields solutions in which copper and iron accompany molybdenum as impurities. The purification of such solutions using the tertiary amine Alamine 336, the quaternary ammonium salt Aliquat 336 or mixtures of both as selective extractants for molybdenum is studied here. Stripping is accomplished with ammonium hydroxide solutions, obtaining ammonium molybdate solutions which can be recirculated to the main circuit for the obtention of ammonium molybdate.
\end{abstract}

Keywords: Solvent extraction. Purification. Molybdenum. Amines. Quaternary ammonium salts.

\section{INTRODUCCIÓN}

El empleo de la extracción con disolventes en la recuperación de molibdeno de disoluciones acuosas se ha aplicado principalmente a la eliminación o separación de este metal de los circuitos de uranio y a la obtención de este último metal de forma más pura.

Además de acompañar al uranio en alguno de sus minerales, el molibdeno se puede encontrar en minerales propios, como la molibdenita, $\mathrm{MoS}_{2}$, o asociado a otros, como la volframita o ciertos minerales cupríferos. Entre otros usos, este metal se utili-

(•) Trabajo recibido el día 4 de mayo de 1995.

${ }^{*}$ Dpto. de Ingeniería Metalúrgica. Facultad de Ingeniería. Universidad de Santiago de Chile. Casilla 10233. Santiago de Chile (Chile).

(**) Centro Nacional de Investigaciones Metalúrgicas (CSIC). Avda. de Gregorio del Amo, 8. 28040-Madrid (España). za como componente de materiales especiales en equipos de alta tecnología, presentando sus procesos convencionales de obtención inconvenientes con respecto a la pureza del producto final, por lo que se han propuesto procesos por vía hidrometalúrgica que, empleando la extracción con disolventes, pueden mejorar este requerimiento de elevada pureza.

En disolución acuosa, el molibdeno presenta distintos estados de oxidación, pudiéndose encontrar en función del valor del $\mathrm{pH}$ de estas disoluciones como especies mono o polinucleares catiónicas o aniónicas, por lo que puede extraerse utilizando los agentes de extracción ácidos, básicos o neutros (1-11).

En el presente trabajo se describe un procedimiento desarrollado para obtener molibdeno puro a partir de un concentrado de molibdenita (12). El esquema simplificado del mismo se muestra en la figura 1. La lixiviación se lleva a cabo en medio $\mathrm{HNO}_{3}$, obteniéndose un residuo rico en $\mathrm{MoO}_{3}$ y una disolución que contiene molibdeno, cobre y 


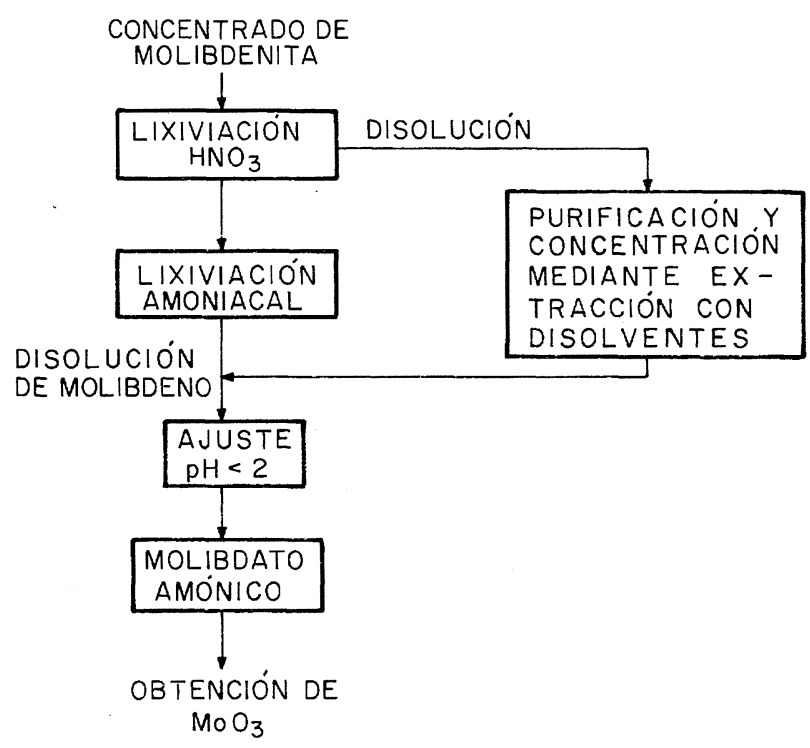

FIG. 1.- Esquema general del tratamiento del concentrado de molibdenita.

FIG. 1.-General scheme for the treatment of the molybdenite concentrate.

hierro, considerándose estos dos últimos elementos como impurezas. Esta disolución se puede recircular, aunque dependiendo de la concentración de molibdeno en la misma se puede pensar en su evaporación y concentración. En ambos casos, se estaría bien recirculando o bien concentrando las impurezas, debiendo tener en cuenta, además, otras consideraciones económicas (por ejemplo, el gasto que supondría la etapa de evaporación). El empleo de la extracción con disolventes permitiría purificar y concentrar el molibdeno y reintegrar este molibdeno residual al circuito principal del proceso.

En el presente trabajo se estudia la aplicación de dos agentes de extracción, la amina terciaria Alamine 336 y la sal de amonio cuaternario Aliquat 336, en la purificación de estas disoluciones de molibdeno.

\section{PARTE EXPERIMENTAL}

Los agentes de extracción Alamine 336 y Aliquat 336, empleados en el presente trabajo, se obtuvieron de Henkel Chile y Henkel Ireland Ltd.; se utilizaron sin purificar, y sus características principales se muestran en la tabla I.

Como diluyente de la fase orgánica se utilizó Escaid 100, de Exxon Chemical Iberia, que presenta las siguientes propiedades: densidad, $805 \mathrm{~kg} / \mathrm{m}^{3}$ (a $15{ }^{\circ} \mathrm{C}$ ), contenido de compuestos aromáticos, 24 $\%$, intervalo de ebullición, $199-237{ }^{\circ} \mathrm{C}$, punto de inflamación, $81^{\circ} \mathrm{C}$. El resto de los reactivos químicos empleados fueron de calidad R.A.

La disolución acuosa empleada en los ensayos de extracción se obtuvo de la lixiviación de un concentrado de molibdenita con ácido nítrico (12); esta disolución presentaba la siguiente composición: 4,3 $\mathrm{g} / \mathrm{l} \mathrm{Mo,} \mathrm{0,28} \mathrm{g/l} \mathrm{Fe,} \mathrm{0,08} \mathrm{g/l} \mathrm{Cu} \mathrm{y} \mathrm{valor} \mathrm{de} \mathrm{pH}$ 0,7.

Los ensayos de extracción-reextracción se llevaron a cabo en embudos de separación termostatizados a la temperatura requerida, provistos de agitación mecánica. El análisis de los elementos metálicos en disolución se realizó mediante espectroscopía de absorción atómica.

Ensayos previos mostraron que en la etapa de extracción, utilizando Alamine 336 o Aliquat 336, se formaban terceras fases: para eliminarlas, se ensayaron como modificadores isodecanol, dodecanol y TBP (tributil fosfato), logrando eliminar con los dos últimos la tercera fase, pero obteniendo con TBP mejores características de coalescencia de los correspondientes sistemas. En general, se hace necesario emplear una relación amina-sal de amonio cuaternario/TBP de 1/1-2, expresada en $\% \mathrm{v} / \mathrm{v}$ en la fase orgánica de cada componente, para obtener buenas separaciones de fases tanto en extracción como en reextracción.

\section{RESULTADOS Y DISCUSIÓN}

\subsection{Extracción}

Se ha estudiado la influencia del tiempo de equilibrado en la extracción de molibdeno mediante estos dos agentes. Las fases orgánicas estaban compuestas por Alamine $336(5 \% \mathrm{v} / \mathrm{v})$-TBP $(5 \% \mathrm{v} / \mathrm{v})$ o Aliquat $336(5 \% \mathrm{v} / \mathrm{v})$-TBP (5\% v/v) en Escaid 100 , mientras que como fase acuosa se empleó la correspondiente disolución descrita en la parte experimental. La temperatura fue de $20^{\circ} \mathrm{C}$ y la

TABLA I.- Características del Alamine 336 y del Aliquat 336

TABLE I.- Alamine 336 and Aliquat 336 characteristics

\begin{tabular}{|l|c|c|c|c|c|}
\hline $\begin{array}{l}\text { Agente de } \\
\text { extracción }\end{array}$ & Tipo & Estructura & $\begin{array}{c}R, \text { cadena } \\
\text { alquílica }\end{array}$ & $\begin{array}{c}\text { Peso } \\
\text { molecular }\end{array}$ & $\begin{array}{c}\text { Densidad, } \\
\mathrm{kg} / \mathrm{m}^{3} \mathrm{a} 25^{\circ} \mathrm{C}\end{array}$ \\
\hline Alamine 336 & Amina terciaria & $\mathrm{R}_{3} \mathrm{~N}$ & $\mathrm{C}_{8-10}$ & 392 & 810 \\
\hline Aliquat 336 & $\begin{array}{l}\text { Sal de amonio } \\
\text { cuaternario }\end{array}$ & $\mathrm{R}_{3} \mathrm{NCH}_{3}^{+} \mathrm{Cl}^{-}$ & $\mathrm{C}_{8-10}$ & 442 & 884 \\
\hline
\end{tabular}


relación de fases O/A igual a 1 . Los resultados se muestran en la figura 2; representan el valor porcentual de extracción de $\mathrm{Mo}(\mathrm{VI})$ frente al tiempo de equilibrado y se observa que, cuando se emplea la sal de amonio cuaternario, el equilibrio de extracción se alcanza entre los 2-3 min de contacto, mientras que con la amina terciaria Alamine 336 son necesarios al menos $5 \mathrm{~min}$ de contacto. En estas condiciones, además, el tanto por ciento de extracción de molibdeno es mayor en el caso del Aliquat 336 que en el caso del Alamine 336.

En la figura 3 se muestra la influencia de la concentración del agente de extracción sobre la extracción de molibdeno. La fase acuosa empleada fue la obtenida en la etapa de lixiviación, utilizándose disoluciones orgánicas que presentaban distintas concentraciones de Alamine 336 o Aliquat 336 y, en cada caso, la misma concentración de TBP (expresada en \% v/v) que del agente de extracción. La temperatura de ensayo fue $20^{\circ} \mathrm{C}$, el tiempo de equilibrado de 6 min y la relación O/A igual a 1 . De esta figura, se deduce que el aumento de la concentración del agente de extracción da lugar a un aumento del tanto por ciento de extracción del molibdeno, resultando más evidente este efecto cuando se comparan, sobre todo, los resultados obtenidos con las concentraciones más bajas de Alamine 336 (1-2 \% v/v) con las más altas.

Para estudiar el efecto del cambio de $\mathrm{pH}$ de la disolución acuosa sobre la extracción de molibdeno y hierro mediante Alamine 336 o Aliquat 336, se ha empleado la disolución acuosa de lixiviación y fases orgánicas de Alamine 336 (5\% v/v)-TBP (5

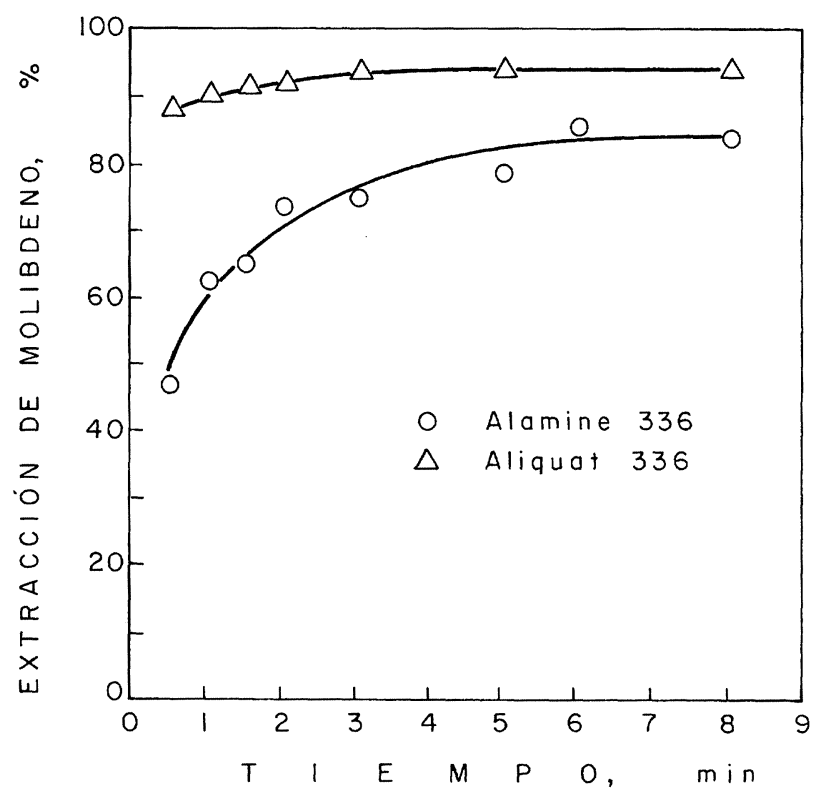

FIG. 2.- Influencia del tiempo de equilibrado en la extracción de molibdeno.

FIG. 2.- Influence of equilibration time on molybdenum extraction.

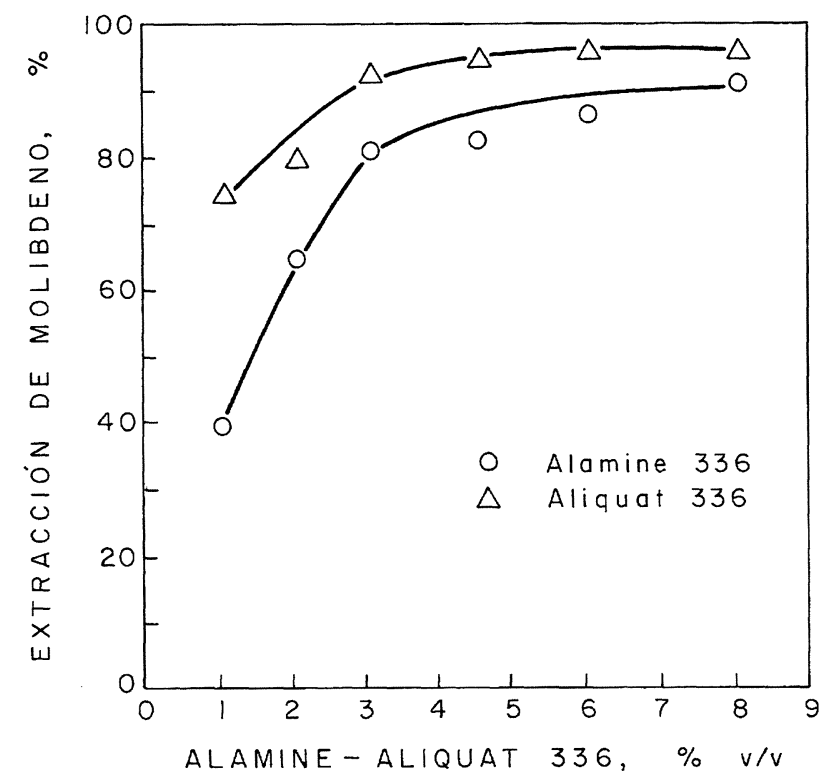

Fig. 3.- Variación del valor porcentual de extracción de molibdeno frente a la concentración del agente de extracción.

FIG. 3.- Variation of the percentage of molybdenum extraction with the extractant concentration.

$\% \mathrm{v} / \mathrm{v})$ y Aliquat $336(5 \% \mathrm{v} / \mathrm{v})-\mathrm{TBP}(5 \% \mathrm{v} / \mathrm{v})$ en Escaid 100. El resto de las condiciones experimentales se mantuvieron iguales a las descritas anteriormente. En la figura 4 se muestra la extracción de ambos metales en función del $\mathrm{pH}$ de equilibrio. En el caso del Aliquat 336, el molibdeno se extrae preferentemente al hierro para el intervalo de valores

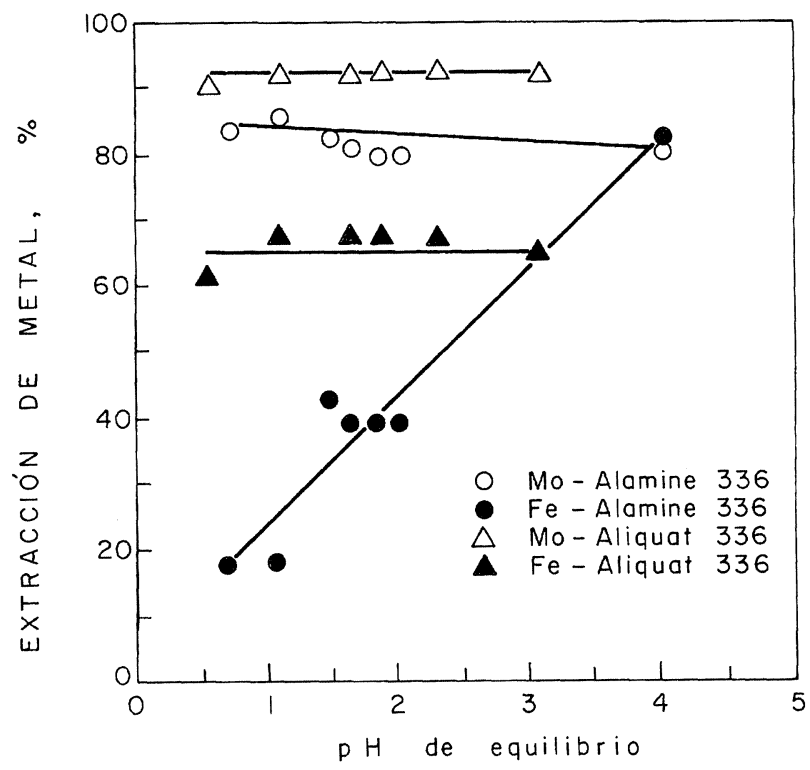

FIG. 4.- Extracción de molibdeno y hierro con el $\mathrm{pH}$ de equilibrio. Temperatura $20,{ }^{\circ} \mathrm{C}$.

FIG. 4.- Molybdenum and iron extraction vs. equilibrium $\mathrm{pH}$. Temperature, $20^{\circ} \mathrm{C}$. 
de $\mathrm{pH}$ obtenidos $(0,5-3,1)$, mientras que en el caso de la amina terciaria Alamine 336, la selectividad con respecto al molibdeno tiende a disminuir a medida que aumenta el valor del $\mathrm{pH}$ de equilibrio. En estos intervalos de $\mathrm{pH}$ obtenidos y en las condiciones experimentales empleadas, el cobre no se extrae ni por el Alamine 336 ni por el Aliquat 336.

Se han obtenido las correspondientes isotermas de equilibrio de extracción de molibdeno con Alamine 336 y Aliquat 336 en Escaid 100. Las fase acuosas y orgánicas empleadas fueron las descritas con anterioridad, así como el resto de las variables experimentales, excepto que en este caso se utilizaron distintas relaciones de fases O/A para la obtención de los puntos experimentales. La figura 5 muestra estas isotermas de extracción, observándose que la carga de molibdeno en la fase orgánica de Aliquat 336 es sensiblemente mayor que la obtenida en el caso del Alamine 336, permitiendo, por lo tanto, el empleo de relaciones A/O más elevadas. Sin embargo, según aumenta el contenido de molibdeno en la fase orgánica la separación de fases comienza a ser peor, hecho que se debe a la formación de precipitados, posiblemente distintos complejos amina/sal de amonio cuaternario-molibdeno. La formación de estos precipitados impide llegar al empleo de relaciones A/O elevadas, siendo, por lo tanto, uno de los inconvenientes de estos sistemas de extracción. Se ha cuantificado cuando aparecen estos complejos precipitados, mostrando las tablas II y III los resultados obtenidos para el caso del Alamine 336 y para el Aliquat 336, respectivamente.

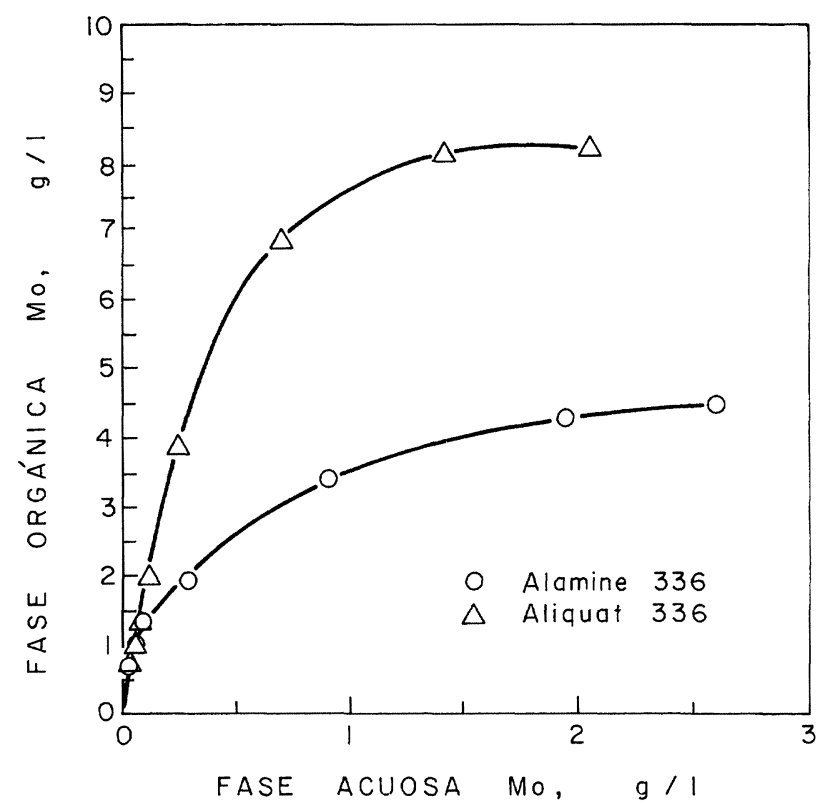

FIG. 5.- Isotermas de equilibrio de extracción de molibdeno. Temperatura, $20^{\circ} \mathrm{C}$.

FIG. 5.- Molybdenum loading equilibrium isotherms. Temperature, $20^{\circ} \mathrm{C}$.
TABLA II.- Influencia de la razón molar $[\mathrm{Mo}]_{\text {org }} /[\text { Alamine } 336]_{\text {org }}$ sobre la obtención de precipitados (complejos amina-Mo(VI))

TABLE II.- Influence of the molar ratio $[M o]_{\text {org }}$ [ [Alamine 336] org on the obtention of precipitates (complexes amine-Mo(VI))

\begin{tabular}{|c|c|}
\hline Razón molar & Precipitación \\
\hline 0,14 & no \\
0,20 & no \\
0,48 & no \\
0,73 & no \\
0,84 & sí \\
1,10 & sí \\
1,24 & sí \\
2,14 & sí \\
\hline
\end{tabular}

TABLA III.- Influencia de la razón molar [Mo] $]_{\text {org }} /[\text { Aliquat 336] }]_{\text {org }}$ sobre la obtención de precipitados (complejos sal de amonio cuaternario$\mathrm{Mo}(\mathrm{VI})$ )

TABLE III.- Influence of the molar ratio [Mo] $]_{\text {org }} /[$ Aliquat 336] org on the obtention of precipitates (complexes quaternary ammonium salt$M o(V I))$

\begin{tabular}{|c|c|}
\hline Razón molar & Precipitación \\
\hline 0,06 & no \\
0,1 & no \\
0,14 & no \\
0,21 & no \\
0,27 & no \\
0,36 & no \\
0,4 & sí \\
0,72 & sí \\
0,82 & sí \\
1,08 & sí \\
2,15 & sí \\
\hline
\end{tabular}

En el caso de la amina terciaria, el mecanismo probable de extracción del molibdeno podría estar representado por la ecuación general:

$$
\begin{gathered}
n R_{3} \mathrm{NH}^{+} \mathrm{NO}_{3 \text { org }}^{-}+\mathrm{Mo}_{\mathrm{a}} \mathrm{O}_{\mathrm{b}} \mathrm{H}_{\text {cac }}^{\mathrm{n}-} \Leftrightarrow\left(R_{3} \mathrm{NH}^{+}\right)_{\mathrm{n}} \\
\mathrm{Mo}_{\mathrm{a}} \mathrm{O}_{\mathrm{b}} \mathrm{H}_{\text {corg }}^{\mathrm{n}-}+n \mathrm{NO}_{3 \mathrm{ac}}^{-}
\end{gathered}
$$

Cuando se emplea la sal de amonio cuaternario Aliquat 336, la reacción de extracción del molibdeno estaría representada por la ecuación general: 


$$
\begin{gathered}
n R_{3} \mathrm{NCH}_{3}^{+} \mathrm{Cl}_{\text {org }}^{-}+\mathrm{Mo}_{\mathrm{a}} \mathrm{O}_{\mathrm{b}} \mathrm{H}_{\mathrm{cac}}^{\mathrm{n}-} \Leftrightarrow\left(R_{3} \mathrm{NCH}_{3}^{+}\right)_{\mathrm{n}} \\
\mathrm{Mo}_{\mathrm{a}} \mathrm{O}_{\mathrm{b}} \mathrm{H}_{\text {corg }}^{\mathrm{n}-}+n \mathrm{Cl}_{\mathrm{ac}}^{-}
\end{gathered}
$$

aunque con este agente de extracción, y empleando otro diluyente de la fase orgánica, se ha descrito que la estequiometría de la especie extraída se puede representar por $\left(R_{3} \mathrm{NCH}_{3}^{+}\right)_{4} \mathrm{Mo}_{8} \mathrm{O}_{26}^{4-}$ e, incluso, con la extracción del ion tetramolibdato (13).

En cualquier caso, parece evidente que, en este medio, el mecanismo de extracción del molibdeno mediante estos dos agentes responde, principalmente, a una reacción de intercambio aniónico.

\subsection{Reextracción}

Teniendo en cuenta el esquema del proceso en el cual se integra la corriente de molibdeno, se empleó exclusivamente como agente de reextracción una disolución de hidróxido amónico.

Las fases orgánicas de Alamine 336-TBP o Aliquat 336-TBP se cargaron respectivamente con 7,5 $\mathrm{g} / \mathrm{l}$ o $10,5 \mathrm{~g} / \mathrm{l}$ de molibdeno, empleando como disolución acuosa inicial la obtenida en la operación de lixiviación del concentrado de molibdenita.

La figura 6 muestra la variación porcentual de reextracción de molibdeno frente a distintos tiempos de contacto. Como disolución acuosa de reextracción, se empleó $1,43 \mathrm{M}$ de $\mathrm{NH}_{4} \mathrm{OH}$ (sistema Alamine 336-TBP) ó $0,86 \mathrm{M}$ de $\mathrm{NH}_{4} \mathrm{OH}$ (sistema Aliquat $336-\mathrm{TBP}$ ), a una temperatura de $20{ }^{\circ} \mathrm{C}$ y

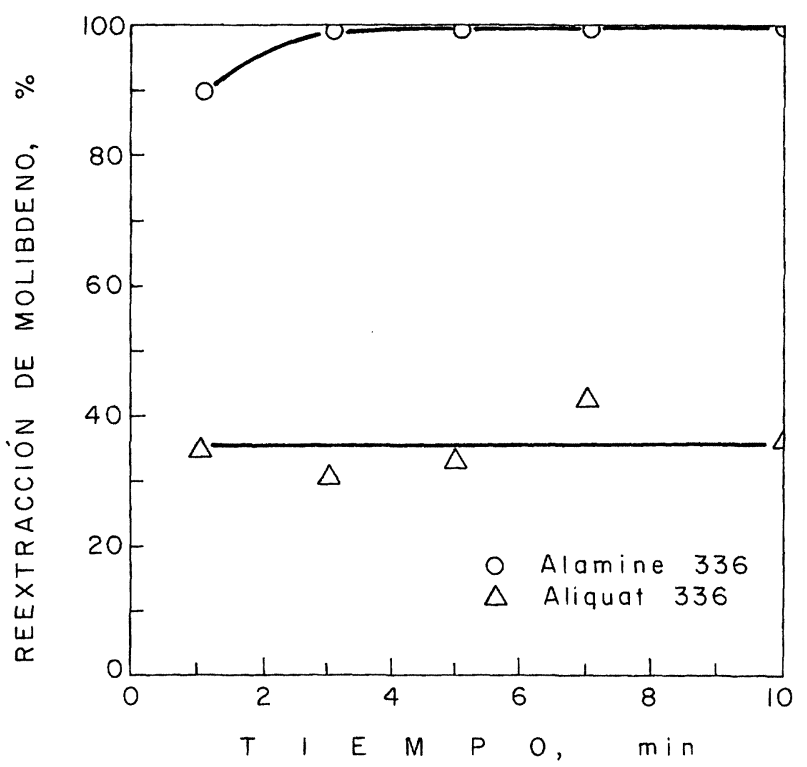

FIG. 6.- Influencia del tiempo de equilibrado en la reextracción de molibdeno.

FIG. 6.- Influence of equilibration time on molybdenum stripping. con relación de fases O/A igual a 3. Se observa que en ambos sistemas el equilibrio se alcanza con rapidez, aproximadamente con 3-5 min de contacto.

El efecto del cambio de la concentración de la disolución de $\mathrm{NH}_{4} \mathrm{OH}$ sobre la reextracción de molibdeno se muestra en la figura 7. En este caso, las condiciones experimentales ensayadas fueron: temperatura $20{ }^{\circ} \mathrm{C}$, tiempo de contacto $10 \mathrm{~min}$ y relación $\mathrm{O} / \mathrm{A}$ igual a 3 . Se observa que la reextracción es más efectiva en el caso del sistema Alamine 336-TBP, aunque el efecto del aumento de la concentración de $\mathrm{NH}_{4} \mathrm{OH}$ sobre la reextracción de molibdeno es contrario; así, en el sistema anteriormente mencionado el tanto por ciento de reextracción del metal tiende inicialmente a disminuir al aumentar la concentración de $\mathrm{NH}_{4} \mathrm{OH}$ para luego permanecer constante, mientras que en el sistema Aliquat 336-TBP la recuperación de molibdeno aumenta al incrementarse la concentración de $\mathrm{NH}_{4} \mathrm{OH}$, aunque a partir de un determinado punto este valor también permanece constante.

En el caśo de este último sistema, se observa que, para concentraciones de $\mathrm{NH}_{4} \mathrm{OH}$ superiores a $1,5 \mathrm{M}$, la disolución acuosa reextraída tiende a precipitar.

La figura 8 muestra las isotermas de reextracción de molibdeno; en el caso del sistema Alamine 336-TBP, se empleó como fase acuosa una disolución $1,43 \mathrm{M}$ de $\mathrm{NH}_{4} \mathrm{OH}$, mientras que para el sistema Aliquat 336-TBP la concentración empleada fue de $0,86 \mathrm{M}$. En ambos casos, la temperatura fue 20 ${ }^{\circ} \mathrm{C}$ y el tiempo de contacto $5 \mathrm{~min}$. En estas condiciones se deduce que se pueden conseguir

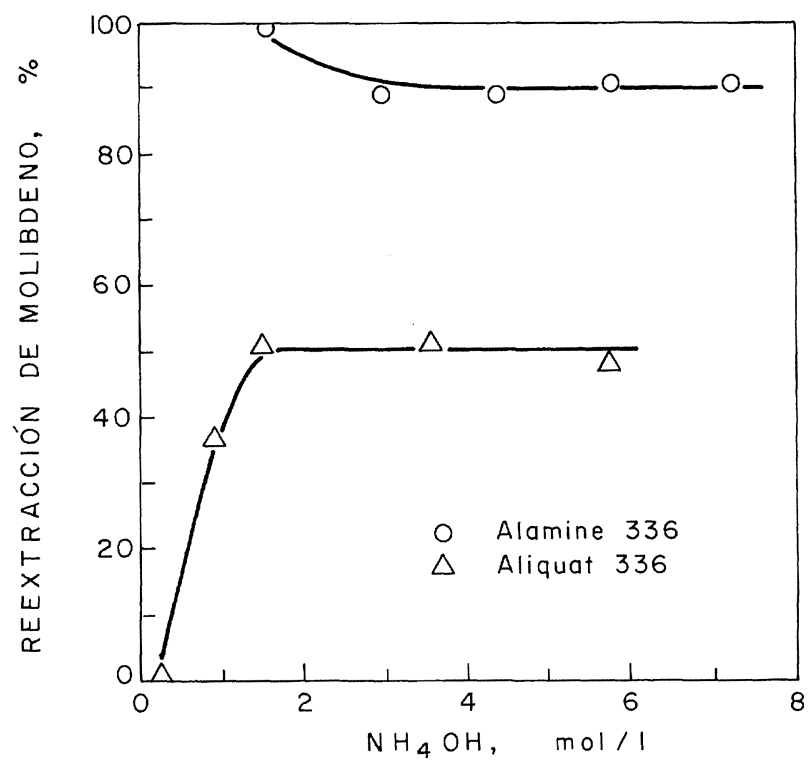

FIG. 7.- Influencia de la concentración de $\mathrm{NH}_{4} \mathrm{OH}$ en la reextracción de molibdeno.

FIG. 7.- Influence of the $\mathrm{NH}_{4} \mathrm{OH}$ concentration on molybdenum stripping. 


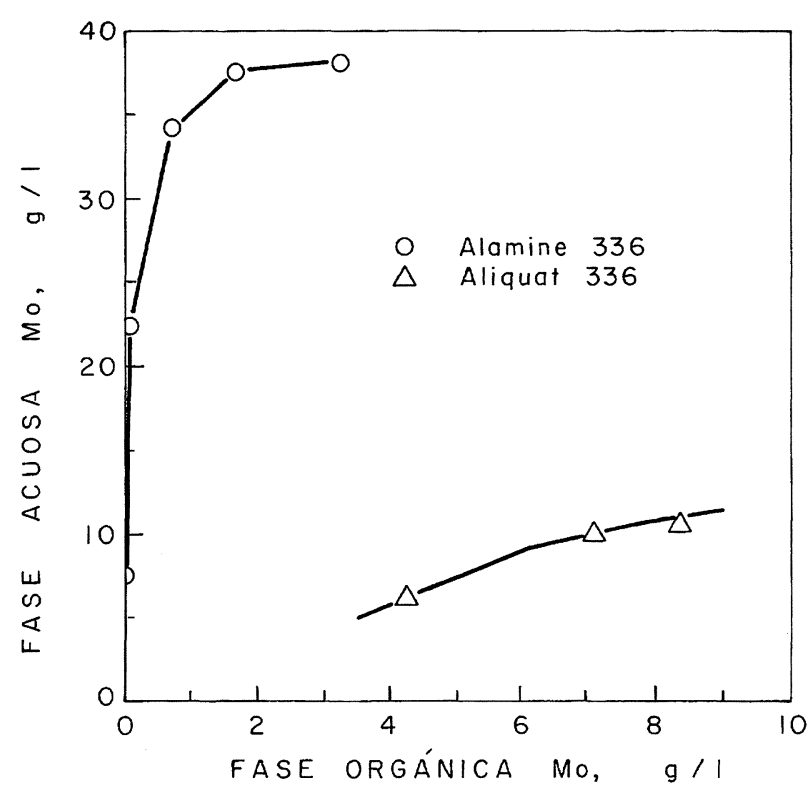

FIG. 8.- Isotermas de reextracción de molibdeno.

FIG. 8.-Molybdenum stripping isotherms.

concentraciones de molibdeno en la disolución acuosa sensiblemente superiores cuando se utiliza el sistema Alamine 336-TBP.

\subsection{Ensayos de extracción-reextracción empleando mezclas Alamine 336-Aliquat 336}

La utilización del Alamine 336 o del Aliquat 336 en la extracción de molibdeno, aunque efectiva en líneas generales, presenta una serie de inconvenientes (por ejemplo, baja recuperación del metal de la fase orgánica cargada en el caso de la sal de amonio cuaternario) por lo que se ha ensayado el empleo de una mezcla de ambos agentes de extracción para estudiar su efecto sobre la extracciónreextracción del molibdeno.

Para el caso de la etapa de extracción, como fase orgánica se empleó una compuesta por Alamine $336(5 \% \mathrm{v} / \mathrm{v})$-Aliquat $336(5 \% \mathrm{v} / \mathrm{v})-\mathrm{TBP}(10 \%$ $\mathrm{v} / \mathrm{v}$ ) en Escaid 100, la disolución acuosa era la correspondiente obtenida en la etapa de lixiviación, es decir, estaba compuesta por 4,3 g/l Mo, 0,28 g/l $\mathrm{Fe}, 0,08 \mathrm{~g} / \mathrm{l} \mathrm{Cu}$ y $\mathrm{pH} 0,7$. La temperatura fue de 20 ${ }^{\circ} \mathrm{C}$ y el tiempo de contacto de 6 min, utilizándose, además, distintas relaciones O/A. La figura 9 muestra la correspondiente isoterma de equilibrio; si se compara ésta con las obtenidas con cada uno de los agentes de extracción por separado (Fig. 5), se observa que con la mezcla de ambos se consigue aumentar la carga de molibdeno en la fase orgánica.

La reextracción del metal también se ha abordado empleando disoluciones de $\mathrm{NH}_{4} \mathrm{OH}$ como fases acuo-

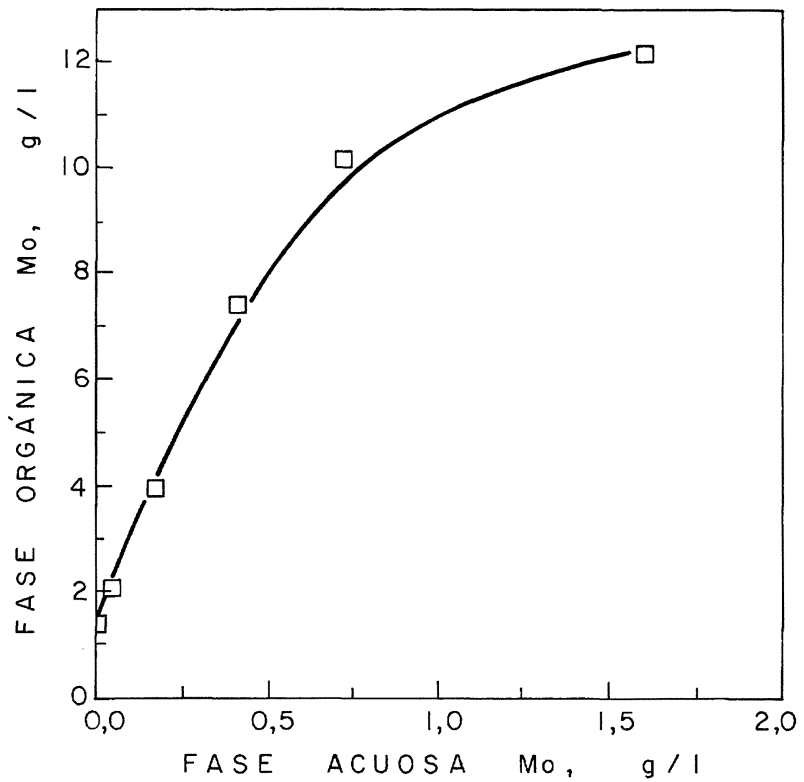

FIG. 9.- Isoterma de equilibrio de extracción de molibdeno para mezcla de Alamine 336-Aliquat 336.

FIG. 9.- Molybdenum loading equilibrium isotherms for Alamine 336-Aliquat 336 mixture.

sas. Las fases orgánicas presentaban la composición mencionada con anterioridad y estaban cargadas con $16,5 \mathrm{~g} / \mathrm{l}$ de molibdeno. En la figura 10 se muestra el

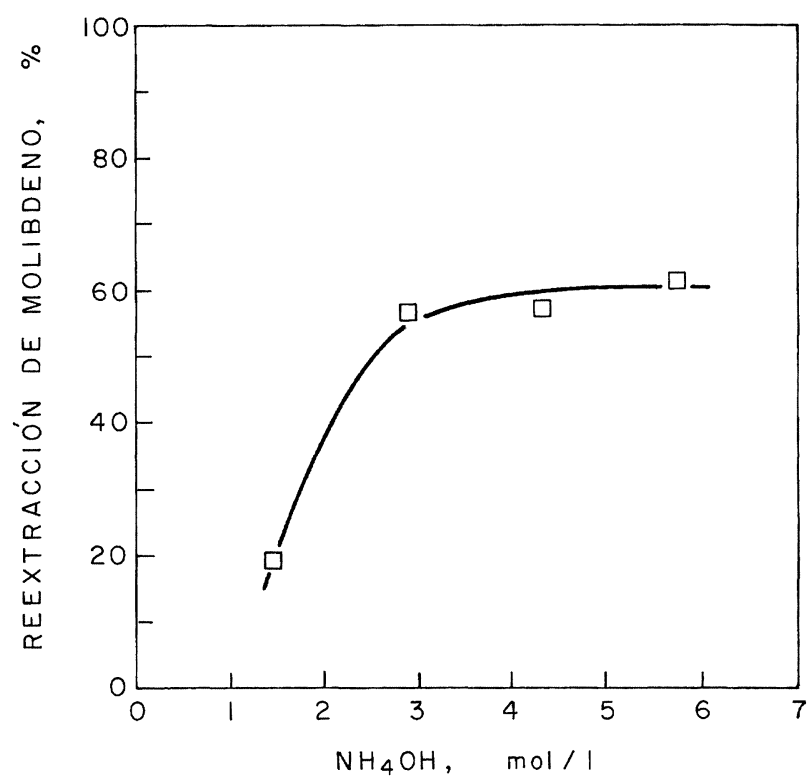

FIG. 10.- Influencia de la concentración de $\mathrm{NH}_{4} \mathrm{OH}$ en la reextracción de molibdeno. Fase orgánica: Alamine 336-Aliquat 336. Temperatura, $20{ }^{\circ} \mathrm{C}$.

FIG. 10.-Influence of the $\mathrm{NH}_{4} \mathrm{OH}$ concentration on molybdenum stripping. Organic phase: Alamine 336-Aliquat 336. Temperature, $20^{\circ} \mathrm{C}$. 
efecto del cambio de la concentración de $\mathrm{NH}_{4} \mathrm{OH}$ sobre la reextracción de molibdeno. Se observa que la recuperación del metal se incrementa al aumentar la concentración del agente de reextracción, aunque para concentraciones $>3 \mathrm{M}$ la reextracción de molibdeno permanece constante. En estas condiciones, no se observa, en ningún caso, la presencia de precipitados en las disoluciones reextraidas.

La figura 11 muestra la isoterma de reextracción obtenida con la fase orgánica anterior y una disolución $2,86 \mathrm{M}$ de $\mathrm{NH}_{4} \mathrm{OH}$, siendo la temperatura de 20 ${ }^{\circ} \mathrm{C}$ y el tiempo de contacto de 5 min con distintas relaciones $\mathrm{O} / \mathrm{A}$.

\section{CONCLUSIONES}

La amina terciaria Alamine 336 extrae al molibdeno de las disoluciones acuosas en medio $\mathrm{NO}_{3}^{-}$, con preferencia al hierro (para valores de $\mathrm{pH}<4$ ) y al cobre. La utilización de esta amina necesita de un modificador para evitar la formación de terceras fases y precipitados en la fase orgánica cargada con molibdeno. Como modificador, se puede utilizar TBP en una relación amina/TBP de 1/1-2, expresadas ambas en $\% \mathrm{v} / \mathrm{v}$ en la fase orgánica. El equilibrio de extracción de molibdeno se alcanza aproximadamente a los 5 min de contacto, no influyendo apreciablemente ni el $\mathrm{pH}$ de equilibrio (para valores comprendidos entre 0,75-4) ni la concentración de Alamine 336 (para concentraciones superiores a $3 \% \mathrm{v} / \mathrm{v}$ ) en la extracción del metal. La reextracción de este metal se puede

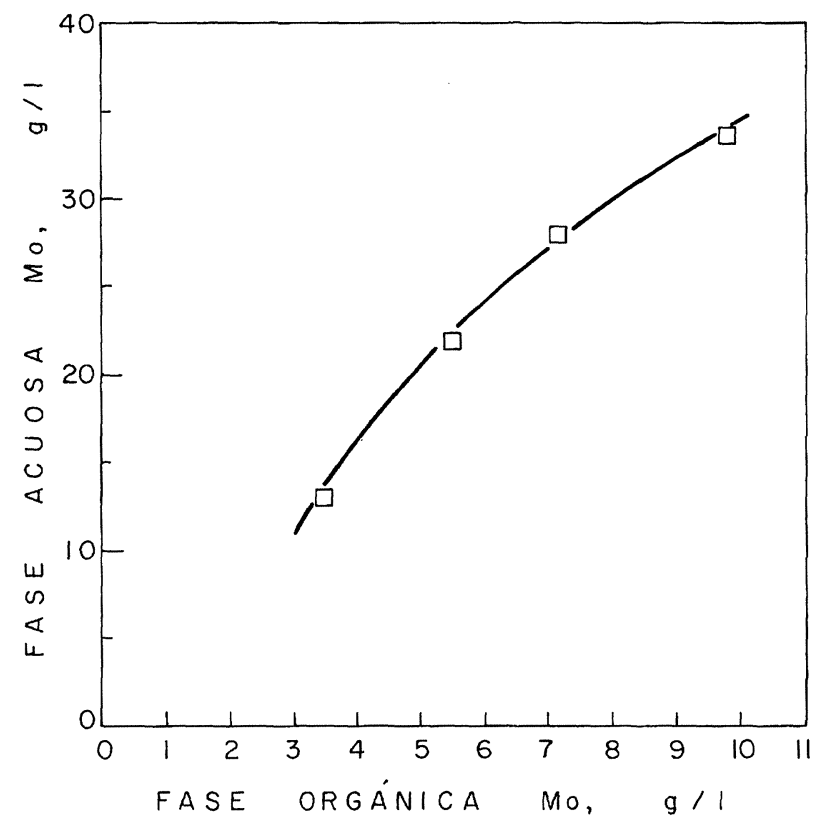

FIG. 11.- Isoterma de reextracción de molibdeno para mezcla de Alamine 336-Aliquat 336.

FIG. 11.-Molybdenum stripping isotherm for Alamine 336-Aliquat 336 mixture. llevar a cabo de forma efectiva empleando disoluciones de hidróxido amónico; el equilibrio de reextracción se alcanza a los 3 min de contacto, aunque si se emplean relaciones de fases O/A superiores a 7/1 la separación de fases empieza a ser mala.

La sal de amonio cuaternario Aliquat 336 es un agente de extracción efectivo del molibdeno de las disoluciones acuosas en medio nitrato, no influyendo el pH sobre la extracción de este metal. Es necesaria la utilización de un modificador en la fase orgánica para evitar la formación de terceras fases y precipitados en la fase orgánica; estos tienden a formarse cuando la relación molar Mo/Aliquat 336 en la fase orgánica es $>0,4$. El equilibrio de extracción se alcanza a partir de los 2 min de contacto. La reextracción está limitada por la presencia de precipitados en la fase acuosa para concentraciones de $\mathrm{NH}_{4} \mathrm{OH}>1,5 \mathrm{M}$.

El empleo de una mezcla de Alamine 336 y Aliquat 336 da lugar a un efecto sinérgico con respecto a la extracción de molibdeno, ya que aumenta la carga del métal en la fase orgánica. Además, se incrementa la concentración de molibdeno en la fase reextraida (respecto al Aliquat 336), puesto que permite llevar a cabo esta etapa con mayores concentraciones de $\mathrm{NH}_{4} \mathrm{OH}$.

\section{Agradecimiento}

Los autores agradecen a la USACH (Chile) y al CSIC (España) la ayuda prestada para la realización de este trabajo.

\section{REFERENCIAS}

(1) Ritcey, G.M. y Ashbrook, A.W. Solvent Extraction. Ed. Elsevier. Amsterdam, 1979: 268-278.

(2) Karagiozov, L. y Vasilev, Ch. Hydrometallurgy, 4, 1979: 51-55.

(3) Karagiozov, L. y Vasilev, Ch. J. Inorg. Nucl. Chem., 43, 1981: 199-200.

(4) AMER, S. Rev. Metal., 18 (4), 1982: 229-244.

(5) Karagiozov, L. y Vasilev, Ch. Hydrometallurgy, 12 , 1984: 111-116.

(6) Lee, T.W., Cheng, W.L. y Ting, G. Solvent Extr. Ion Exch., 2, 1984: 435-450.

(7) Sato, T., Watanabe, H. y Suzuki, H. Solvent Extr. Ion Exch., 4, 1986: 987-998.

(8) WanG, X., Yang, Z., QiaO, X., Wangaud, Y. y Yan, J. Proc. First International Conference on Hydrometallurgy (ICHM '88). Eds. Y. Zheng y J. Xu. Int. Academic Pub. Pekín, 1988: 180-184.

(9) Sato, T., Watanabe, H. y Suzuki, H. Hydrometallurgy, 23, 1990: 297-308.

(10) CocA, J., Diez, F.V. y Moris, M.A. Hydrometallurgy, 25, 1990: 125-135.

(11) Zhu, T. Hydrometallurgy, 27, 1991: 231-245.

(12) Navarro, P. y Alguacil, F.J. Hydrometallurgy. En revisión, 1995.

(13) Bal, Y., Cote, G. y Bauer, D. Solvent Extraction 1990. Ed. T. Sekine. Elsevier. Amsterdam, 1992: 919-924. 